Simon Sium - Rama Shanker

\title{
A zero-truncated discrete Akash distribution with properties and applications*
}

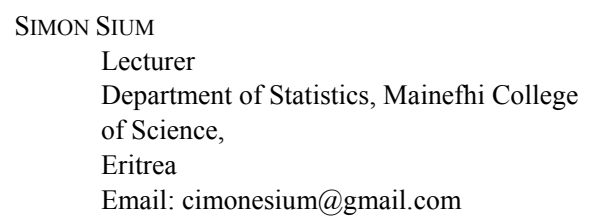

Email: cimonesium@gmail.com

\author{
RAMA SHANKER \\ Associate Professor \\ Department of Statistics, Assam University \\ Silchar, \\ India \\ Email: shankerrama2009@gmail.com
}

This study proposes and examines a zero-truncated discrete Akash distribution and obtains its probability and moment-generating functions. Its moments and moments-based statistical constants, including coefficient of variation, skewness, kurtosis, and the index of dispersion, are also presented. The parameter estimation is discussed using both the method of moments and maximum likelihood. Applications of the distribution are explained through three examples of real datasets, which demonstrate that the zero-truncated discrete Akash distribution gives better fit than several zero-truncated discrete distributions.

KEYWORDS: zero-truncated distribution, discrete Akash distribution, goodness of fit

A zero-truncated version of the original discrete distribution having probability mass function (pmf) $P_{0}(x ; \theta)$ can be defined as

$$
P(x ; \theta)=\frac{P_{0}(x ; \theta)}{1-P_{0}(0 ; \theta)} ; x=1,2,3, \ldots
$$

In probability theory, zero-truncated distributions are certain discrete distributions whose support is the set of positive integers. Berhane-Shanker [2018a] have

* The authors are grateful for the comments given by the editor-in-chief and the reviewers to improve the quality and presentation of the paper.

HungaRian StaTISTICAL REVIEW, VOLUME 3, NUMBER 2, PP. 12-25. DOI: 10.35618/hsr2020.02.en012 
recently introduced the discrete Akash distribution (DAD), a discrete analogue of Shanker's [2015] continuous Akash distribution based on an infinite series approach of discretisation. DAD is defined by its pmf:

$$
P_{0}(x ; \theta)=\frac{\left(e^{\theta}-1\right)^{3}}{e^{\theta}\left(e^{2 \theta}-e^{\theta}+2\right)}\left(1+x^{2}\right) e^{-\theta x} ; x=0,1,2,3, \ldots, \theta>0 .
$$

Berhane-Shanker [2018a] obtained moments and moments-based measures of DAD and showed that both the method of moments and that of maximum likelihood give the same estimator of the parameter $\theta$. Further, the authors discussed its applications to model count data from biological sciences and demonstrated that it gives better fit than Poisson and Poisson-Lindley distributions. Introduced by Shanker [2015], the Akash distribution is defined by its probability density function (pdf):

$$
f_{1}(x ; \theta)=\frac{\theta^{3}}{\theta^{2}+2}\left(1+x^{2}\right) e^{-\theta x} ; x>0, \theta>0 .
$$

Shanker [2015] provides detailed discussion about its various mathematical and statistical properties, estimation of parameters, and application for modelling waiting time. The Lindley distribution proposed by Lindley [1958] is defined by its pdf:

$$
f_{2}(x ; \theta)=\frac{\theta^{2}}{\theta+1}(1+x) e^{-\theta x} ; x>0, \theta>0 .
$$

Various statistical properties, estimation of parameters, and application to model waiting time data in a bank are available in Ghitany-Atieh-Nadarajah [2008]. Shanker-Hagos-Sujatha [2015a] conducted a detailed comparative study of applications of exponential and Lindley distributions for modelling lifetime data from various fields of knowledge and observed that both are competing with each other. Berhane-Shanker [2018b] presented a discrete version of Lindley distribution, that is, discrete Lindley distribution (DLD), using an infinite approach of discretisation defined by its pmf:

$$
P_{1}(x ; \theta)=\frac{\left(e^{\theta}-1\right)^{2}}{e^{2 \theta}}(1+x) e^{-\theta x} ; x=0,1,2,3, \ldots, \theta>0 .
$$

Simon-Shanker [2018] derived zero-truncated discrete Lindley distribution (ZTDLD) corresponding to DLD defined by its pmf:

HungaRian StaTisticAL REVIEW, VOluME 3, NuMBER 2, PP. 12-25. DOI: 10.35618/hsr2020.02.en012 


$$
P_{2}(x ; \theta)=\frac{\left(e^{\theta}-1\right)^{2}}{2 e^{\theta}-1}(1+x) e^{-\theta x} ; x=1,2,3, \ldots, \theta>0 .
$$

Statistical properties, estimation of parameters, and applications of DLD and ZTDLD are discussed in Berhane-Shanker [2018b] and Simon-Shanker [2018].

Shanker [2017a] presented a Poisson-Akash distribution (PAD), which is a Poisson mixture of Akash distribution. Similarly, Sankaran [1970] suggested a Poisson-Lindley distribution (PLD), which is a Poisson mixture of Lindley [1958] distribution. Ghitany-Al-Mutairi-Nadarajah [2008] and Shanker [2017b] respectively proposed the zero-truncated versions of PLD and PAD known as zero-truncated Poisson-Lindley distribution (ZTPLD) and zero-truncated Poisson-Akash distribution (ZTPAD). Shanker et al. [2015b] conducted a comparative study on applications of zero-truncated Poisson distribution (ZTPD) and ZTPLD. The pmf of PLD, PAD, ZTPLD and ZTPAD are presented in Table 1.

Table 1

Pmf of PLD, PAD, ZTPLD and ZTPAD

\begin{tabular}{l|c}
\hline \multicolumn{1}{c}{ Name } & Distribution \\
\hline PLD & $P(x ; \theta)=\frac{\theta^{2}(x+\theta+2)}{(\theta+1)^{x+3}} ; x=0,1,2, \ldots, \theta>0$ \\
\hline PAD & $P(x ; \theta)=\frac{\theta^{3}}{\theta^{2}+2} \cdot \frac{x^{2}+3 x+\left(\theta^{2}+2 \theta+3\right)}{(\theta+1)^{x+3}} ; x=0,1,2,3, \ldots, \theta>0$ \\
\hline ZTPLD & $P(x ; \theta)=\frac{\theta^{2}}{\theta^{2}+3 \theta+1} \cdot \frac{x+\theta+2}{(\theta+1)^{x}} ; x=1,2,3, \ldots, \theta>0$ \\
\hline ZTPAD & $P(x ; \theta)=\frac{\theta^{3}\left\{x^{2}+3 x+\left(\theta^{2}+2 \theta+3\right)\right\}}{\left(\theta^{4}+2 \theta^{3}+7 \theta^{2}+6 \theta+2\right)(\theta+1)^{x}} ; x=1,2,3, \ldots, \theta>0$ \\
\hline
\end{tabular}

Shanker et al. [2015b] conducted an extensive study on the comparison between ZTPD and ZTPLD with respect to their applications in datasets excluding zero-counts and found that in demography and biological sciences ZTPAD gives better fit than ZTPD, while in social sciences ZTPD gives better fit than ZTPLD.

This study suggests a zero-truncated discrete Akash distribution (ZTDAD) by taking the zero-truncated version of DAD introduced by Berhane-Shanker [2018a]. Its generating functions and moments about the origin and the mean are obtained.

HungaRian StatisticAl ReVIEW, VOluME 3, NuMBER 2, PP. 12-25. DOI: 10.35618/hsr2020.02.en012 
Furthermore, this study presents expressions for coefficient of variation $(\mathrm{CV})$, skewness $\left(\sqrt{\beta_{1}}\right)$, kurtosis $\left(\beta_{2}\right)$ and the index of dispersion $(\gamma)$. The parameter estimation of ZTDAD is also discussed. Finally, applications of ZTDAD to three observed real datasets are used to test its goodness of fit over ZTPD, ZTPLD, ZTPAD, and ZTDLD.

\section{A zero-truncated discrete Akash distribution}

Using $/ 1 /$ and $/ 2 /$, the pmf and cumulative function $[F(t ; \theta)]$ of ZTDAD can be obtained as

$$
\begin{gathered}
P_{3}(x ; \theta)=\frac{\left(e^{\theta}-1\right)^{3}}{2 e^{2 \theta}-e^{\theta}+1}\left(1+x^{2}\right) e^{-\theta x} ; x=1,2,3, \ldots, \theta>0, \\
F(t ; \theta)=1-\frac{e^{-\theta(t-2)}\left(t^{2}+2 t+2\right)-e^{-\theta(t-1)}\left(2 t^{2}+2 t+1\right)+e^{-\theta t}\left(t^{2}+1\right)}{2 e^{2 \theta}-e^{\theta}+1} ; \\
t=1,2,3, \ldots, \theta>0 .
\end{gathered}
$$

Figure 1. Behaviour of ZTDAD for varying values of parameter $\theta$

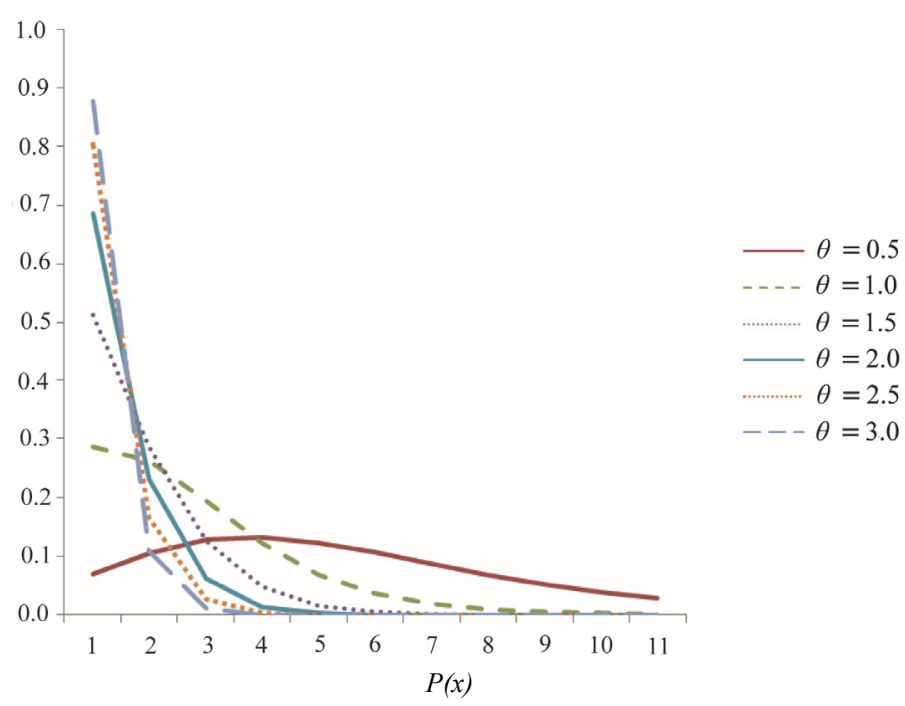

Hungarian Statistical ReVIEW, Volume 3, NumBER 2, PP. 12-25. DOI: 10.35618/hsr2020.02.en012 
Since $\frac{P_{3}(x+1 ; \theta)}{P_{3}(x ; \theta)}=\frac{1}{e^{\theta}}\left(1+\frac{2 x+1}{x^{2}+1}\right)$ is a decreasing function of $x>0$, $P_{3}(x ; \theta)$ is log-concave. Therefore, ZTDAD is unimodal, has an increasing failure rate, and hence an increasing failure rate average. It is new better than used, new better than used in expectation, and has decreasing mean residual life. Detailed discussions and interrelationships between these ageing concepts are available in Barlow-Proschan [1981].

\section{Moments and moments-based measures}

The probability-generating function $[G(t)]$ and moment-generating function $[M(t)]$ of ZTDAD can be obtained as

$$
G(t)=\frac{\left(e^{\theta}-1\right)^{3}}{\left(2 e^{2 \theta}-e^{\theta}+1\right)} \frac{\left(2 t e^{2 \theta}-t^{2} e^{\theta}+t^{2}\right)}{\left(e^{\theta}-t\right)^{3}} \text { for } t \neq e^{\theta}
$$

and

$$
M(t)=\frac{\left(e^{\theta}-1\right)^{3}}{\left(2 e^{2 \theta}-e^{\theta}+1\right)} \frac{\left(2 e^{2 \theta}-e^{\theta+t}+e^{2 t}\right)}{\left(e^{\theta}-e^{t}\right)^{3}} \text { for } t \neq \theta .
$$

It can be easily verified that the function in /9/ is infinitely differentiable with respect to $t$ since it involves exponential terms of its argument. This means that all moments about the origin $\mu_{r}^{\prime}, r \geq 1$ of ZTDAD can be obtained. The first four moments about the origin of ZTDAD can be obtained as

$$
\begin{gathered}
\mu_{1}^{\prime}=\frac{2 e^{\theta}\left(e^{2 \theta}+e^{\theta}+1\right)}{\left(2 e^{2 \theta}-e^{\theta}+1\right)\left(e^{\theta}-1\right)}, \\
\mu_{2}^{\prime}=\frac{2 e^{\theta}\left(e^{3 \theta}+5 e^{2 \theta}+5 e^{\theta}+1\right)}{\left(2 e^{2 \theta}-e^{\theta}+1\right)\left(e^{\theta}-1\right)^{2}},
\end{gathered}
$$

Hungarian Statistical ReVIEW, Volume 3, NumBER 2, PP. 12-25. DOI: 10.35618/hsr2020.02.en012 


$$
\begin{gathered}
\mu_{3}^{\prime}=\frac{2 e^{\theta}\left(e^{4 \theta}+14 e^{3 \theta}+30 e^{2 \theta}+14 e^{\theta}+1\right)}{\left(2 e^{2 \theta}-e^{\theta}+1\right)\left(e^{\theta}-1\right)^{3}}, \\
\mu_{4}^{\prime}=\frac{2 e^{\theta}\left(e^{5 \theta}+33 e^{4 \theta}+146 e^{3 \theta}+146 e^{2 \theta}+33 e^{\theta}+1\right)}{\left(2 e^{2 \theta}-e^{\theta}+1\right)\left(e^{\theta}-1\right)^{4}} .
\end{gathered}
$$

The relationship between moments about the origin and the mean gives the moments about the mean of ZTDAD as

$$
\begin{gathered}
\mu_{2}=\sigma^{2}=\frac{2 e^{\theta}\left(5 e^{4 \theta}-2 e^{2 \theta}+2 e^{\theta}+1\right)}{\left(2 e^{2 \theta}-e^{\theta}+1\right)^{2}\left(e^{\theta}-1\right)^{2}}, \\
\mu_{3}=\frac{2 e^{\theta}\left(10 e^{7 \theta}+15 e^{6 \theta}-42 e^{5 \theta}+47 e^{4 \theta}+2 e^{3 \theta}-15 e^{2 \theta}+6 e^{\theta}+1\right)}{\left(2 e^{2 \theta}-e^{\theta}+1\right)^{3}\left(e^{\theta}-1\right)^{3}}, \\
\mu_{4}=\frac{2 e^{\theta}\left(\begin{array}{l}
20 e^{10 \theta}+270 e^{9 \theta}-307 e^{8 \theta}+216 e^{7 \theta}+356 e^{6 \theta}-548 e^{5 \theta}+ \\
+402 e^{4 \theta}-48 e^{3 \theta}-24 e^{2 \theta}+22 e^{\theta}+1
\end{array}\right)}{\left(2 e^{2 \theta}-e^{\theta}+1\right)^{4}\left(e^{\theta}-1\right)^{4}} .
\end{gathered}
$$

Finally, $C V, \sqrt{\beta_{1}}, \beta_{2}$ and $\gamma$ of ZTDAD are obtained as

$$
\begin{gathered}
C V=\frac{\sigma}{\mu_{1}^{\prime}}=\frac{\sqrt{2 e^{\theta}\left(5 e^{4 \theta}-2 e^{2 \theta}+2 e^{\theta}+1\right)}}{2\left(e^{3 \theta}+e^{2 \theta}+e^{\theta}\right)}, \\
\sqrt{\beta_{1}}=\frac{\mu_{3}}{\left(\mu_{2}\right)^{3 / 2}}=\frac{2 e^{\theta}\left(10 e^{7 \theta}+15 e^{6 \theta}-42 e^{5 \theta}+47 e^{4 \theta}+2 e^{3 \theta}-15 e^{2 \theta}+6 e^{\theta}+1\right)}{\left\{2 e^{\theta}\left(5 e^{4 \theta}-2 e^{2 \theta}+2 e^{\theta}+1\right)\right\}^{3 / 2}}
\end{gathered}
$$




$$
\begin{gathered}
\beta_{2}=\frac{\mu_{4}}{\mu_{2}{ }^{2}}=\frac{\left(\begin{array}{l}
20 e^{10 \theta}+270 e^{9 \theta}-307 e^{8 \theta}+216 e^{7 \theta}+356 e^{6 \theta}-548 e^{5 \theta}+ \\
+402 e^{4 \theta}-48 e^{3 \theta}-24 e^{2 \theta}+22 e^{\theta}+1
\end{array}\right)}{2 e^{\theta}\left(5 e^{4 \theta}-2 e^{2 \theta}+2 e^{\theta}+1\right)^{2}}, \\
\gamma=\frac{\sigma^{2}}{\mu_{1}^{\prime}}=\frac{\left(5 e^{4 \theta}-2 e^{2 \theta}+2 e^{\theta}+1\right)}{\left(2 e^{2 \theta}-e^{\theta}+1\right)\left(e^{\theta}-1\right)\left(e^{2 \theta}+e^{\theta}+1\right)} .
\end{gathered}
$$

Table 2 summarises the behaviour of the mean, variance, $C V, \sqrt{\beta_{1}}, \beta_{2}$ and $\gamma$ of ZTDAD for some selected values of parameter $\theta$.

Descriptive statistics of ZTDAD for varying values of parameter $\theta$

\begin{tabular}{c|c|c|c|c|c|c}
\hline$\theta$ & Mean & Variance & $C V$ & $\sqrt{\beta_{1}}$ & $\beta_{2}$ & $\gamma$ \\
\hline 0.2 & 14.83 & 75.68 & 0.59 & 1.22 & 4.96 & 5.10 \\
\hline 0.4 & 7.23 & 19.12 & 0.60 & 1.33 & 4.93 & 2.65 \\
\hline 0.6 & 4.68 & 8.46 & 0.62 & 1.39 & 4.97 & 1.81 \\
\hline 0.8 & 3.42 & 4.65 & 0.63 & 1.45 & 5.11 & 1.36 \\
\hline 1.0 & 2.69 & 2.86 & 0.63 & 1.51 & 5.34 & 1.06 \\
\hline 1.2 & 2.23 & 1.88 & 0.62 & 1.58 & 5.66 & 0.84 \\
\hline 1.4 & 1.91 & 1.30 & 0.60 & 1.67 & 6.06 & 0.68 \\
\hline 1.6 & 1.69 & 0.93 & 0.57 & 1.77 & 6.53 & 0.55 \\
\hline 1.8 & 1.53 & 0.68 & 0.54 & 1.89 & 7.07 & 0.44 \\
\hline 2.0 & 1.42 & 0.51 & 0.50 & 2.03 & 7.71 & 0.36 \\
\hline 2.2 & 1.33 & 0.39 & 0.47 & 2.19 & 8.46 & 0.29 \\
\hline 2.4 & 1.26 & 0.30 & 0.43 & 2.36 & 9.35 & 0.24 \\
\hline
\end{tabular}

It is clear from Table 2 that the mean, variance, $C V$ and $\gamma$ of ZTDAD are decreasing for increasing values of parameter $\theta$, while $\sqrt{\beta_{1}}$ and $\beta_{2}$ of ZTDAD are increasing. The one interesting characteristics of ZTDAD is that $\sigma^{2}>\mu_{1}^{\prime}$ is always over-dispersed $\left(\sigma^{2}>\mu_{1}^{\prime}\right)$ for $\theta \leq 1$ and under-dispersed $\left(\sigma^{2}<\mu_{1}^{\prime}\right)$ for $\theta>1$. This means that ZTDAD is a suitable model for both over- and under-dispersed data according to the value of parameter $\theta \leq 1$ or $\theta>1$. Figure 2 presents the behaviour of $C V, \sqrt{\beta_{1}}, \beta_{2}$ and $\gamma$ of ZTDAD for varying values of parameter $\theta$. 
Figure 2. Behaviour of the coefficient of variation, skewness, kurtosis and the index of dispersion of ZTDAD for varying values of parameter $\theta$
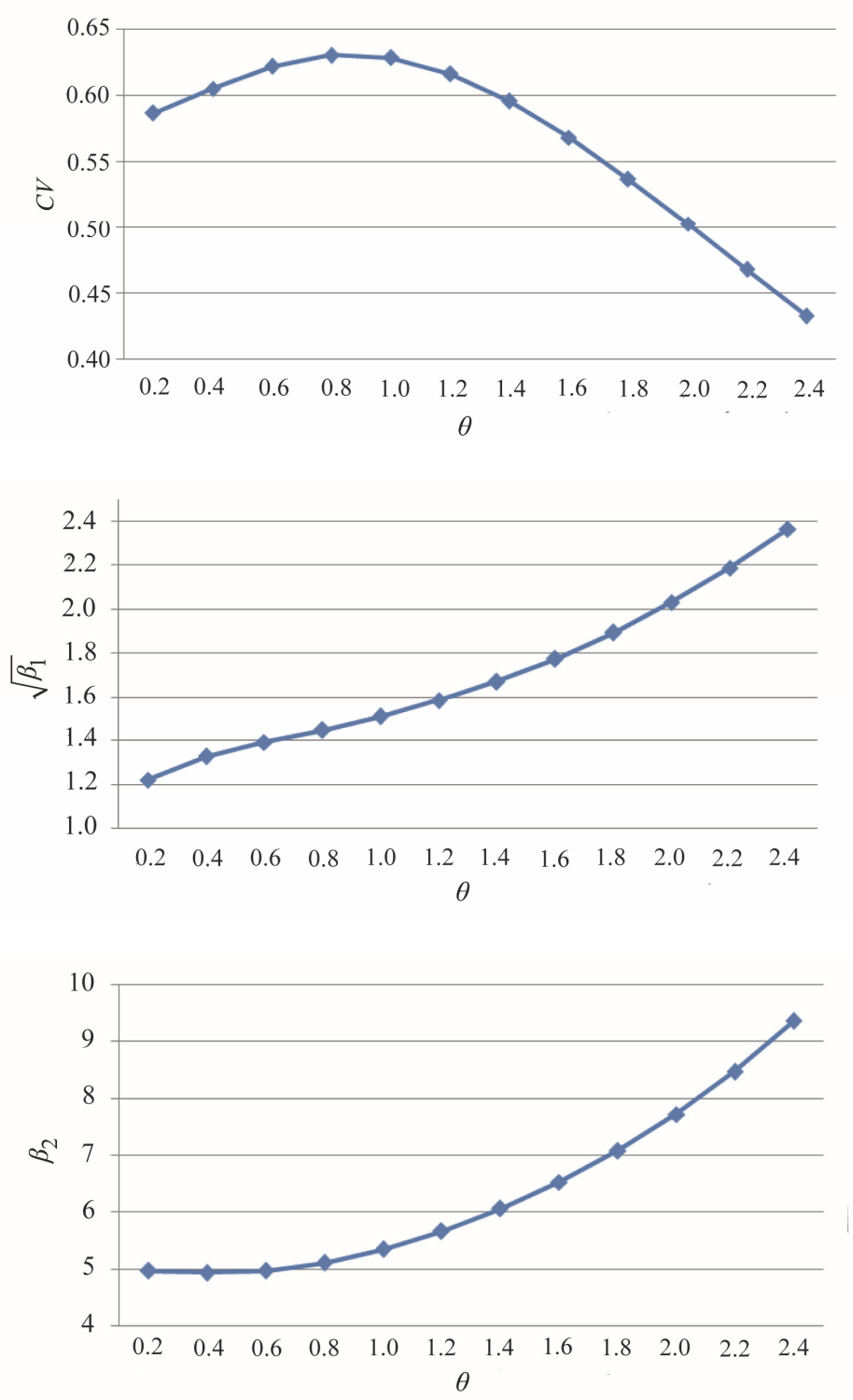

(Continued on the next page) 
(Continued)

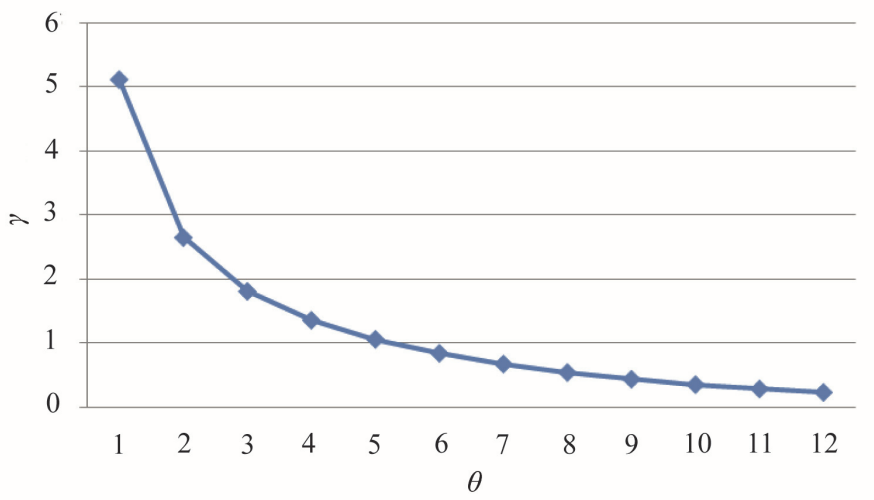

The conditions under which ZTDAD, ZTDLD, and ZTPLD are over-, equi-, and under-dispersed are presented in Table 3.

Table 3

\begin{tabular}{l|c|c|c}
\multicolumn{4}{c}{ Over-, equi- and under-dispersion of ZTDAD, ZTDLD and ZTPLD } \\
\hline $\begin{array}{c}\text { Type of } \\
\text { distribution }\end{array}$ & $\begin{array}{c}\text { Over-dispersion } \\
\left(\mu<\sigma^{2}\right)\end{array}$ & $\begin{array}{c}\text { Equi-dispersion } \\
\left(\mu=\sigma^{2}\right)\end{array}$ & $\begin{array}{c}\text { Under-dispersion } \\
\left(\mu>\sigma^{2}\right)\end{array}$ \\
\hline ZTDAD & $\theta<1.0514$ & $\theta=1.0514$ & $\theta>1.0514$ \\
\hline ZTDLD & $\theta<0.8612$ & $\theta=0.8612$ & $\theta>0.8612$ \\
\hline ZTPLD & $\theta<1.2586$ & $\theta=1.2586$ & $\theta>1.2586$ \\
\hline
\end{tabular}

Figure 3. Over-, equi- and under-dispersion of ZTDAD

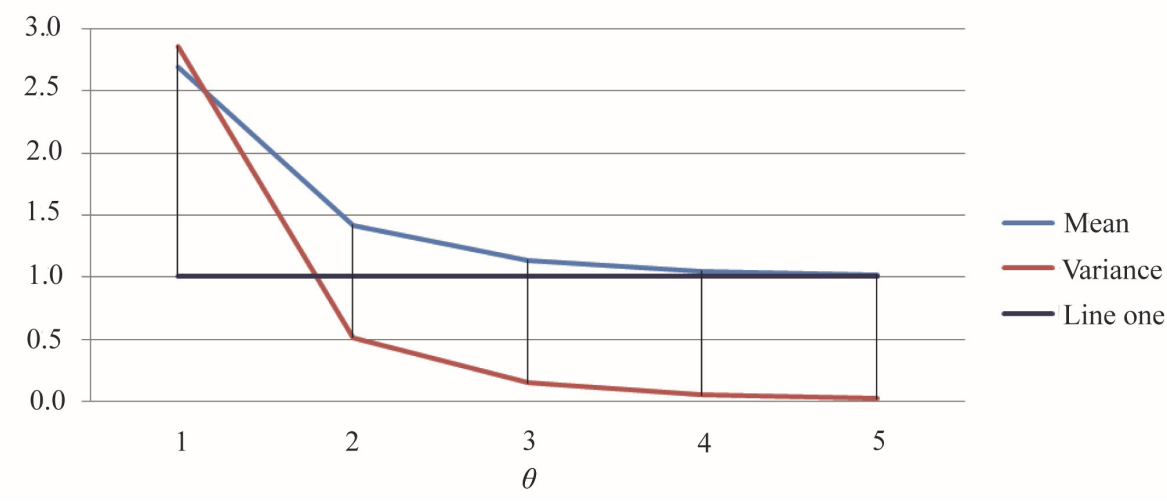

Hungarian Statistical Review, Volume 3, NuMBER 2, PP. 12-25. DOI: 10.35618/hsr2020.02.en012 


\section{Parameter estimation}

Method of moments estimate (MOME). Let $x_{1}, x_{2}, \ldots, x_{n}$ be a random sample of size $n$ from ZTDAD /7/. Equating the population mean to the corresponding sample mean, MOME $\tilde{\theta}$ of $\theta$ is the solution of the following non-linear equation:

$$
2(1-\bar{x}) e^{3 \theta}+(2+3 \bar{x}) e^{2 \theta}+2 e^{\theta}(1-\bar{x})+\bar{x}=0
$$

where $\bar{x}$ is the sample mean.

Maximum likelihood estimate (MLE): Let $x_{1}, x_{2}, \ldots, x_{n}$ be a random sample of size $n$ from ZTDAD /7/. The likelihood function $L$ of ZTDAD /7/ is given by

$$
L=\left(\frac{\left(e^{\theta}-1\right)^{3}}{2 e^{2 \theta}-e^{\theta}+1}\right)^{n} \prod_{i=1}^{n}\left(1+x_{i}^{2}\right) e^{-\theta x_{i}} .
$$

The log likelihood function is given by

$$
\ln L=n\left\{3 \ln \left(e^{\theta}-1\right)-\ln \left(2 e^{2 \theta}-e^{\theta}+1\right)\right\}+\sum_{i=1}^{n} \ln \left(1+x_{i}^{2}\right)-n \theta \bar{x}
$$

The MLE $\hat{\theta}$ of the parameter $\theta$ is the solution of the following log likelihood equation:

$$
\frac{d \ln L}{d \theta}=\frac{3 n e^{\theta}}{e^{\theta}-1}-\frac{4 n e^{2 \theta}-e^{\theta}}{2 e^{2 \theta}-e^{\theta}+1}-n \bar{x}=0
$$

which gives $2(1-\bar{x}) e^{3 \theta}+(2+3 \bar{x}) e^{2 \theta}+2 e^{\theta}(1-\bar{x})+\bar{x}=0$. This means that both the method of moments and maximum likelihood give the same estimate of the parameter of ZTDAD. This non-linear equation can be solved by any numerical iteration method such as Newton-Raphson, Bisection, and Regula-Falsi methods. The present study used the Newton-Raphson method. 


\section{Applications}

ZTDAD has been fitted to a number of real datasets to test its goodness of fit over ZTPD, ZTPLD, ZTPAD, and ZTDLD. Based on the results, it provides better fit in most cases. Maximum likelihood estimation of the parameter has been used to fit other distributions. This study presents three examples of real datasets, two from biological sciences and one from demography. The first dataset is the number of counts of flower heads per the number of fly eggs reported by Finney-Varley [1955]. (See Table 4.) The second dataset is a collection of data corresponding to the number of snowshoe hares captured over seven days and reported by Keith-Meslow [1968]. (See Table 5.) The third dataset includes data on the number of leaf spots of mulberry variety 'Ichinose', reported in Khurshid [2008].

It is obvious from the Chi-square $\left(\chi^{2}\right)$ and $p$-values that ZTDAD gives much closer fit than ZTPD, ZTPLD, ZTPAD, and ZTDLD. Therefore, ZTDAD can be considered an important tool for modelling count data excluding zero-count over the others.

Table 4

Number of counts of flower heads per number of fly eggs

\begin{tabular}{|c|c|c|c|c|c|c|}
\hline \multirow{2}{*}{$\begin{array}{l}\text { Number of } \\
\text { fly eggs }\end{array}$} & \multirow{2}{*}{$\begin{array}{l}\text { Observed } \\
\text { frequency }\end{array}$} & \multicolumn{5}{|c|}{ Expected value } \\
\hline & & ZTPD & ZTPLD & ZTPAD & ZTDLD & ZTDAD \\
\hline 1 & 22 & 15.3 & 26.8 & 25.1 & 24.9 & 20.7 \\
\hline 2 & 18 & 21.9 & 19.8 & 19.8 & 20.4 & 21.1 \\
\hline 3 & 18 & 20.8 & 13.9 & 14.7 & 14.9 & 17.3 \\
\hline 4 & 11 & 14.9 & 9.5 & 10.3 & 10.2 & 12.2 \\
\hline 5 & 9 & 8.5 & 6.4 & 6.9 & 6.7 & 7.52 \\
\hline 6 & 6 & 4.1 & 4.2 & 4.4 & 4.3 & 4.4 \\
\hline 7 & 3 & 1.7 & 2.7 & 2.8 & 2.7 & 2.4 \\
\hline 8 & 0 & 0.6 & 1.7 & 1.7 & 1.6 & 1.3 \\
\hline 9 & 1 & 0.2 & 3.0 & 2.3 & 2.3 & 1.1 \\
\hline Total & 88 & 88.0 & 88.0 & 88.0 & 88.0 & 88.0 \\
\hline MLE & & $\hat{\theta}=2.8604$ & $\hat{\theta}=0.7186$ & $\hat{\theta}=1.0215$ & $\hat{\theta}=0.6042$ & $\hat{\theta}=0.8940$ \\
\hline$\chi^{2}$ & & 6.677 & 3.743 & 2.090 & 2.257 & 0.998 \\
\hline$d f$ & & 4 & 4 & 4 & 4 & 4 \\
\hline$p$-value & & 0.1540 & 0.4419 & 0.7192 & 0.7192 & 0.9627 \\
\hline
\end{tabular}

Source: Own calculation based on Finney-Varley [1955].

Hungarian STATISTICAL REVIEW, VOluME 3, NuMBER 2, PP. 12-25. DOI: 10.35618/hsr2020.02.en012 
Table 5

Number of snowshoe hares captured over seven days

\begin{tabular}{l|c|c|c|c|c|c}
\hline \multirow{2}{*}{$\begin{array}{c}\text { Number of } \\
\text { hares captured }\end{array}$} & \multirow{2}{*}{$\begin{array}{c}\text { Observed } \\
\text { frequency }\end{array}$} & \multicolumn{5}{|c}{ Expected value } \\
\cline { 3 - 7 } & & ZTPD & ZTPLD & ZTPAD & ZTDLD & ZTDAD \\
\hline 1 & 122 & 115.8 & 124.7 & 124.4 & 125.1 & 119.8 \\
\hline 2 & 50 & 57.4 & 46.7 & 47.0 & 48.4 & 52.5 \\
\hline 3 & 18 & 18.9 & 17.0 & 17.2 & 16.7 & 18.3 \\
\hline 5 & 4 & 4.7 & 6.1 & 6.1 & 5.4 & 5.5 \\
\hline MLE & 4 & 1.2 & 3.5 & 3.3 & 2.4 & 1.9 \\
\hline$\chi^{2}$ & 198 & 198.0 & 198.0 & 198.0 & 198.0 & 198.0 \\
\hline$d f$ & & $\hat{\theta}=0.9906$ & $\hat{\theta}=2.1830$ & $\hat{\theta}=2.6140$ & $\hat{\theta}=1.3189$ & $\hat{\theta}=1.7420$ \\
\hline$p$-value & & 2.140 & 0.617 & 0.460 & 0.243 & 0.209 \\
\hline
\end{tabular}

Source: Own calculation based on Keith-Meslow [1968].

Table 6

Leaf spot grades of mulberry variety 'Ichinose'

\begin{tabular}{l|c|c|c|c|c|c}
\hline \multirow{2}{*}{ Leaf spot grade } & \multirow{2}{*}{$\begin{array}{c}\text { Observed } \\
\text { frequency }\end{array}$} & \multicolumn{5}{|c}{ Expected value } \\
\cline { 3 - 7 } & & ZTPD & ZTPLD & ZTPAD & ZTDLD & ZTDAD \\
\hline 1 & 18 & 14.2 & 23.0 & 21.7 & 21.5 & 18.3 \\
\hline 2 & 15 & 18.7 & 16.3 & 16.5 & 16.9 & 17.7 \\
\hline 3 & 10 & 16.5 & 11.1 & 11.6 & 11.8 & 13.7 \\
\hline 5 & 14 & 10.9 & 7.3 & 7.8 & 7.7 & 9.0 \\
\hline MLE & 13 & 9.7 & 12.3 & 12.4 & 12.1 & 11.3 \\
\hline$\chi^{2}$ & 70 & 70.0 & 70.0 & 70.0 & 70.0 & 70.0 \\
\hline$d f$ & & $\hat{\theta}=2.6400$ & $\hat{\theta}=0.7819$ & $\hat{\theta}=1.0565$ & $\hat{\theta}=1.3189$ & $\hat{\theta}=0.9498$ \\
\hline$p$-value & & 6.311 & 7.476 & 5.943 & 6.296 & 4.445 \\
\hline
\end{tabular}

Note. Leaves having 1-5, 6-10, 11-15, 16-20, or more than 20 spots were given grade $1,2,3,4$, or 5, respectively.

Source: Own calculation based on Khurshid [2008]. 


\section{Conclusion}

This study introduced a ZTDAD. Furthermore, the analysis examined the $C V, \sqrt{\beta_{1}}, \quad \beta_{2}$ and $\gamma$ of ZTDAD, as well as their behaviours. Both the method of moments and of maximum likelihood give the same estimates of the parameter of ZTDAD. Three examples of real datasets were presented to test the goodness of fit of ZTDAD over ZTPD, ZTPLD, ZTPAD, and ZTDLD. The results revealed that ZTDAD gives much closer fit over these distributions. Therefore, ZTDAD can be considered an important one-parameter discrete distribution to model count datasets that structurally excludes zero-counts.

\section{References}

Barlow, R. E. - Proschan, F. [1981]: Statistical Theory of Reliability and Life Testing. To Begin With. Silver Spring.

Berhane, A. - Shanker, R. [2018a]: A discrete Akash distribution with applications in biological sciences. Turkiye Klinikleri Journal of Biostatistics. Vol. 10. No. 1. pp. 1-12. https://doi.org/10.5336/biostatic.2017-59265

Berhane, A. - ShanKer, R. [2018b]: A discrete Lindley distribution with applications in biological sciences. Biometrics and Biostatistics International Journal. Vol. 7. No. 2. pp. 48-52. https://doi.org/10.15406/bbij.2018.07.00189

FinNey, D. J. - VARLEY, G. C. [1955]: An example of the truncated Poisson distribution. Biometrics. Vol. 11. No. 3. pp. 387-394. https://doi.org/10.2307/3001776

Ghitany, M. E. - Al-Mutairi, D. K. - Nadarajah, S. [2008]: Zero-truncated Poisson-Lindley distribution and its applications. Mathematics and Computers in Simulation. Vol. 79. No. 3. pp. 279-287. https://doi.org/10.1016/j.matcom.2007.11.021

Ghitany, M. E. - Atieh, B. - NAdARAJAh, S. [2008]: Lindley distribution and its application. Mathematics Computing and Simulation. Vol. 78. Issue 4. pp. 493-506. https://doi.org/10.1016/j.matcom.2007.06.007

Keith, L. B. - Meslow, E. C. [1968]: Trap response by snowshoe hares. Journal of Wildlife Management. Vol. 32. No. 4. pp. 795-801. https://doi.org/10.2307/3799555

Khursid, A. M. [2008]: On size-biased Poisson distribution and its use in zero-truncated case. JKSIAM. Vol. 12. No. 3. pp. 153-160.

LindLEY, D. V. [1958]: Fiducial distributions and Bayes' theorem. Journal of the Royal Statistical Society, Series B. Vol. 20. Issue 1. pp. 102-107. https://doi.org/10.1111/j.25176161.1958.tb00278.x

SanKaran, M. [1970]: The discrete Poisson-Lindley distribution. Biometrics. Vol. 26. No. 1. pp. 145-149. https://doi.org/10.2307/2529053

Hungarian StatistiCAL ReVIEW, VOluME 3, NuMBER 2, PP. 12-25. DOI: 10.35618/hsr2020.02.en012 
SHANKer, R. - Hagos, F. - Sujatha, S. [2015a]: On modeling of lifetimes data using exponential and Lindley distributions. Biometrics and Biostatistics International Journal. Vol. 2. No. 5. pp. 1-9. https://doi.org/10.15406/bbij.2015.02.00042

Shanker, R. - Hagos, F. - Sujatha, S. - Abrehe, Y. [2015b]: On zero-truncation of Poisson and Poisson-Lindley distributions and their applications. Biometrics \& Biostatistics International Journal. Vol. 2. No. 6. pp. 1-14. https://doi.org/10.15406/bbij.2015.02.00045

SHANKER, R. [2015]: Akash distribution and its applications. International Journal of Probability and Statistics. Vol. 4. No. 3. pp. 65-75. https://doi.org/10.5923/j.ijps.20150403.01

SHANKer, R. [2017a]: The discrete Poisson-Akash distribution. International Journal of Probability and Statistics. Vol. 6. No. 1. pp. 1-10. https://doi.org/10.5923/ j.ijps.20170601.01

Shanker, R. [2017b]: Zero-truncated Poisson-Akash distribution and its applications. American Journal of Mathematics and Statistics. Vol. 7. No. 6. pp. 227-236.

SiMON, S. - SHANKER, R. [2018]: A zero-truncated discrete Lindley distribution with applications. International Journal of Statistics in Medical and Biological Sciences. Vol. 2. No. 1. pp. 8-15.

HungaRian StaTisticAL REVIEW, VOluME 3, NuMBER 2, PP. 12-25. DOI: 10.35618/hsr2020.02.en012 